\title{
Cellular Magnetic Resonance Imaging: Nanometer and Micrometer Size Particles for Noninvasive Cell Localization
}

\author{
Jonathan R. Slotkin, ${ }^{1}$ Kevin S. Cahill,*1 Suzanne A. Tharin,* and Erik M. Shapiro ${ }^{\dagger}$ \\ *Department of Neurosurgery, The Brigham and Women's Hospital, The Children's Hospital, Boston, Harvard Medical School, \\ Boston, Massachusetts 02115, and Department of Diagnostic Radiology, Yale University School of Medicine, New Haven, \\ Connecticut 06510
}

\begin{abstract}
Summary: The use of nanometer and micrometer-sized superparamagnetic iron oxide particles as cellular contrast agents allows for the noninvasive detection of labeled cells on highresolution magnetic resonance images. The development and application of these techniques to neurologic disorders is likely to accelerate the development of cell transplantation therapies and allow for the detailed study of in vivo cellular biology. This review
\end{abstract}

\section{INTRODUCTION}

Several promising cellular transplantation therapies for CNS diseases and injury are currently entering human clinical trials. Such experimental therapies include neural stem cells, olfactory ensheathing cells, Schwann cells, and activated macrophages. For example, activated macrophages were recently tested in a Phase II clinical trial by direct injection into the spinal cord of humans with acute spinal cord injury (ProCord; Proneuron Biotechnologies, Los Angeles, CA). ${ }^{1}$ Even as promising cellular treatments for CNS injury and disease rapidly move forward, however, there are no noninvasive, objective methods in current clinical use that allow for the identification and tracking of such cells once they have been transplanted.

There have been rapid advances in the field of imaging technology in the past decade, especially in the areas of cellular and molecular imaging research. One particularly exciting concept uses nanometer- and micrometersized iron oxide particles to label individual cells in vitro for subsequent in vivo MRI. This concept is founded on the principle that nanometer-sized crystals of iron oxide

\footnotetext{
${ }^{1}$ Both authors contributed equally to this work.

Address correspondence and reprint requests to: Erik M. Shapiro, Department of Diagnostic Radiology, Yale University School of Medicine, New Haven, CT 06510. E-mail: erik.shapiro@yale.edu.
}

summarizes the early development of iron oxide-based cellular contrast agents and the more recent application of this technology to noninvasive imaging of cellular transplants. The ability of this technique to allow for the noninvasive detection of in vivo transplants on the single-cell level is highlighted. Key Words: Stem cells, magnetic resonance imaging, single-cell imaging, superparamagnetic contrast agents, iron oxide, brain. 


\section{BACKGROUND}

To identify and track single cells and transplanted cell populations, most investigations have focused on techniques using a contrast agent that can alter the $\mathrm{T}_{1}, \mathrm{~T}_{2}$, and $\mathrm{T}_{2} *$ relaxivities of voxels containing cells of interest. This has been achieved through the application of existing MRI contrast agents, ${ }^{16}$ and also through the application or development of novel contrast agents. Materials such as iron, nickel, cobalt, and gadolinium possess permanent magnetic moments that can produce intense local magnetic fields, ${ }^{17}$ and such substances have been explored as cellular contrast agents.

Iron oxide-containing particles are superparamagnetic and are commonly used as cell-labeling contrast agents. When superparamagnetic particles are placed in an external magnetic field, the magnetic dipoles orient to produce local disruptions in the main magnetic field. This effect, manifested as spin dephasing, influences neighboring protons at a distance equivalent to thousands of times the actual size of the particles themselves. MRI protocols that are sensitive to these dephasing effects elicit $\mathrm{T}_{2} *$ contrast and can be used to detect these particles. $^{17-19}$

\section{LABELING CELL POPULATIONS WITH IRON OXIDE COMPOUNDS}

To distinguish single cells or cellular transplants from host tissue, cells of interest can be tagged or loaded with intracellular magnetic resonance (MR) contrast agents through a variety of means. Initial efforts loaded Tlymphocytes with superparamagnetic particles through an endocytosis-mediated mechanism. ${ }^{20}$ One published technique resulted in $0.11 \mathrm{ng} \mathrm{Fe} / \mathrm{cell}$ and allowed for the detection of $\sim 2 \times 10^{6}$ cells per $30 \mu \mathrm{L}$ of fluid. ${ }^{21}$ Enhanced uptake of superparamagnetic material by $\mathrm{T}$ cells was later achieved by coating the superparamagnetic particles with the transactivator (Tat) protein of the human immunodeficiency virus, which enhanced cellular uptake. ${ }^{10,11}$

Other strategies for labeling cells of interest have relied on the synthesis of mAbs linked to superparamagnetic particles. When the superparamagnetic agent MION-46L was linked to a mAb targeted to the transferrin receptor, enhanced MION-46L uptake could be achieved in a population of oligodendrocyte progenitor cells. ${ }^{9}$ These early efforts suffered from the relative inefficiencies of the labeling processes, the cell-type-specific nature of the Tat protein or $\mathrm{mAb}$ techniques, and the complex schemes required to synthesize such labeling agents.

Less specific but more efficient cellular labeling strategies were later developed. One approach used a superparamagnetic cell-labeling agent called the magnetodendrimer
(MD-100). ${ }^{4}$ This compound consists of carboxylated poly(amidoamine) dendrimers encapsulating a superparamagnetic iron oxide core. Such dendrimers have a known affinity for cellular membranes and have been previously used to transport foreign DNA into cells.

It was hypothesized that the dendrimers could also effectively load cells with iron oxide. ${ }^{22-26}$ The simple addition of MD-100 to cell-culture media for 48 hours resulted in the intracellular accumulation of iron oxide in a variety of cell types, including the HeLa cell line, 3T3 fibroblasts, and oligodendrocyte progenitor cells. ${ }^{4,27}$ This labeling process resulted in a relaxation enhancement five times greater than that achieved using mAbs to the transferrin receptor. This labeling technique was successfully implemented to consecutively monitor the biodistribution of oligodendroglial progenitor transplants into demyelinated rat brain at $4.7 \mathrm{~T}$ and $1.5 \mathrm{~T}$, and has been applied to other organ transplants, including muscle. ${ }^{4,28}$

More recent nanometer-scale techniques have used commercially available cellular transfection reagents combined with ferumoxide as the cellular MR contrast agent. ${ }^{29,30}$ Ferumoxide (Feridex; Advanced Magnetics, Cambridge, MA) is a commercially available SPIO that readily forms complexes with cellular transfection agents such as poly-L-lysine. Such complexes are nonspecifically taken up into endosomes by many cell types and allow for efficient magnetic labeling. ${ }^{7,31}$

\section{TOWARD SINGLE-CELL IMAGING}

As we have noted, cellular detection using intracellular contrast agents has been extensively studied both in vitro and in vivo over the past 15 years. The majority of this work has focused on imaging of transplanted and host cell populations. More recent work has focused on the ability of this technology to allow for single-cell detection by MRI.

Single-cell detection using MRI has been evaluated both in vitro and in vivo. Early work with USPIOs evaluated the sensitivity of MRI at the cellular level. ${ }^{32}$ This work used dextran-coated USPIO particles with an average diameter of $30 \mathrm{~nm}$ to nonspecifically label rat $\mathrm{T}$ cells. Although this work was limited by the inefficient uptake of USPIOs by cultured T cells, images of in vitro SPIO-labeled T cells on microscope slides were obtained at $7 \mathrm{~T}$. Using colabeling with the cellular membrane dye DiI, colocalization of red fluorescence with areas of decreased MR signal corresponding to single cells was illustrated. Electron microscopy studies revealed that the $\mathrm{T}$ cells endocytosed the SPIO particles into larger clusters, creating a localized magnetic field larger than that of the single particles.

The application of USPIO-labeled cells for in vivo cellular detection has also been extensively studied. The detection of small numbers of cells and the migration of 
those cells in the rat brain was illustrated using USPIOlabeled stem cell transplants. ${ }^{33}$ This study used embryonic stems cells transfected with a USPIO contrast agent. Initial in vitro studies of labeled cell phantoms showed that as few as 40 cells could be detected at $7 \mathrm{~T}$, and in vivo studies of stereotactically placed, labeled cell grafts into rat brain showed that cell grafts of as few as 500 cells could be detected in the rat brain. Additionally, the migration pattern of SPIO-labeled, green fluorescent protein (GFP)-producing embryonic stem cells implanted contralateral to an ischemic hemisphere could be followed with serial MRI scans. The migration of the cell grafts toward the ischemic hemisphere was imaged and correlated with the results from conventional histologic GFP studies.

The application of SPIO to single-cell imaging has not been limited to high field research magnets. Other work has shown that single-cell sensitivity is possible using clinical scanners. ${ }^{34}$ SPIO-labeled THP-1 cells were imaged in cell phantoms using a three-dimensional (3D) FIESTA [fast imaging employing steady state acquisition] protocol at a resolution of $100 \mu \mathrm{m}$ isotropic on a 1.5-T clinical whole-body scanner. Using customized high-power gradient coils, individual cells could be detected on 3D FIESTA sequences and colocalized with fluorescent images.

A more recent study from the same group using a 1.5-T clinical scanner demonstrated single-cell detection in mouse brain in vivo. ${ }^{35}$ An intracardiac injection of SPIO-labeled J774 cells was used to deliver cells to the mouse brain. The $\mathrm{J} 774$ cell line is a macrophage cell line capable of obtaining an extremely high 60.9 pg Fe per cell after SPIO labeling. After arterial injection of 10,000 cells, 3D FIESTA sequence MRI scans could detect on average 28 signal voids corresponding to labeled cells in brain. This number corresponds with the predicted number of cells expected in the brain after arterial delivery $(<0.2 \%)$. The areas of decreased signal correlated with DiI fluorescence on confocal microscopy. The demonstrated adaptation of clinical scanners for single-cell scanning holds great promise for the ultimate goal of translating single-cell MRI to human applications. The authors noted that the $60 \mathrm{pg}$ of iron per cell was two to three times higher than the amount of iron accumulated in nonphagocytic cell lines following SPIO labeling. ${ }^{35}$

\section{MPIOS AND SINGLE-CELL IMAGING}

In contrast to nanometer-sized USPIOs, larger superparamagnetic particles in the micrometer size range (MPIOs) have been developed and evaluated for cellular imaging. Microspheres from Bangs Laboratories (Fishers, IN) consist of magnetite iron oxide with a green fluorescent component embedded in a polystyrene matrix. The application of these particles as cellular contrast agents has been directly compared to nanometer-sized particles. ${ }^{36}$ That is, micrometer-sized $(0.9 \mu \mathrm{m}$ average size $)$ superparamagnetic microspheres were directly compared to ferumoxide USPIOs (Feridex: $10 \mathrm{~nm}$ crystal core, $150 \mathrm{~nm}$ overall size).

It was shown in vitro that the MPIOs had increased $\mathrm{R}_{2}$ * relaxivities at an equal iron content, compared with the nanometer size particles $\left(240 \mathrm{mmol} / \mathrm{L}^{-1} \mathrm{~s}^{-1}\right.$ versus $\left.356 \mathrm{mmol} / \mathrm{L}^{-1} \mathrm{~s}^{-1}\right) .{ }^{36}$ This increased relaxation produced a greater degree of hypointensity on MR images. This study also evaluated the labeling efficiency of hematopoetic and mesenchymal stem cells and showed efficient MPIO labeling with incubations as short as 1 hour. Notably, no effect was found on in vitro cellular differentiation assays. Finally, the authors showed that single, labeled cells could be detected in MR images of culture dishes containing live cells at $11.7 \mathrm{~T} .^{36,37}$

In addition to single-cell detection in vitro, recent work using MPIOs has shown that the disruption in magnetic field homogeneity can be detected even from single MPIO particles. ${ }^{38}$ The dark MR signal produced by MPIO particles is robust enough that single MPIOs could be detected at a resolution of $100 \mu \mathrm{m}$. Comparison studies of increasing particle sizes from $0.76 \mu \mathrm{m}$ to 1.63 $\mu \mathrm{m}$ revealed, as expected, increasing contrast with increasing the size of the particles. This study further documented the application of MPIOs to ex vivo single-cell detection in whole organisms. Single-cell mouse embryos were injected with MPIOs then re-implanted and allowed to develop. Fixed embryonic day 11.5 embryos were imaged at 7 T. Punctuate dark areas in single cells could be detected and Prussian blue-stained histology sections confirmed the presence of the detected single MPIOs. $^{38}$

Single-cell detection both in vitro and ex vivo has been demonstrated using cells labeled with micrometer-sized superparamagnetic iron oxide. The application of this technology to obtain images of cells in vivo has also been explored. A recent study applied this technology to a model of hepatocyte transplantation. ${ }^{39}$ Mouse hepatocytes were labeled with $1.63-\mu \mathrm{m}$-diameter MPIOs and injected into the spleen. Labeled hepatocytes that migrated and engrafted into liver were detectable as punctate dark contrast spots on $\mathrm{T}_{2}{ }^{*}$ gradient recalled echo (GRE) images at $7 \mathrm{~T}$. The colabeling of the cells with CM-DiI allowed for detailed histologic studies colocalizing the MPIO particles with CM-DiI-labeled cells.

Additionally, this report included several important control studies examining the fate of MPIO labeling from transplanted cells that died on transplantation and failed to engraft. ${ }^{39}$ Heat-killed MPIO-labeled cells were injected and corresponding MR images of the liver were obtained. In comparison to live injected cells, which produced punctate areas of contrast, the contrast ob- 
served in the liver after transplantation of heat-killed cells was grainy and less intense. Histologic analyses revealed that some free particles could travel from the spleen to the liver, accounting for the grainy contrast seen on MR images; however, careful analyses of signal intensities revealed that signal decreases from labeled cells could be distinguished from the noise-like signal profiles of free particles. This study concluded that single transplanted hepatocytes that migrated from the spleen to engraft in the liver could be detected on in vivo MRI studies and distinguished from residual free label from nonviable cells. ${ }^{39}$

Recent work in mouse brain imaging has revealed that single MPIO-labeled cells can be detected in vivo following intravascular delivery of labeled cells to the brain. ${ }^{35}$ For this study, the human breast carcinoma MDA-MB-231 cell line was labeled with 0.9- $\mu \mathrm{m}$ MPIOs and then delivered to mouse brain by arterial injection. This cell line has a propensity for the formation of brain metastases. Single, labeled cells could be detected on 3D FIESTA MR images at sites that later formed metastases in the mouse brain. The authors showed that this technique can be used to follow the fate of individual cells in the brain over time.

In contrast to the injection and subsequent imaging of MPIO-labeled cells, an alternative strategy is to image cells after direct injection of free MPIOs. The premise is that, because single particles can be detected, inefficient labeling schemes could be tolerated and still yield robust detection of cells. This strategy was applied in rodent brains to demonstrate the ability to obtain MR images of in vivo cell migration of neural progenitor cells along the rostral migratory stream. ${ }^{40}$ Free $1.63-\mu$ m-diameter MPIOs were injected into the lateral ventricles of Sprague-Dawley rats and then imaged at $11.7 \mathrm{~T}$ or $7.0 \mathrm{~T}$. Signal hypointensity could be detected along the rostral migratory stream beginning at 1 week post injection, and persisting at least 5 weeks post injection. Prussian bluestained histology and fluorescent immunohistochemistry sections revealed that astrocytes, ependymal cells, migrating neuroblasts, and mature neurons contained MPIOs. It was hypothesized that labeled neuroblasts differentiated into mature granular interneurons accounting for the labeled neurons. Additionally, cells that presumably died along the migratory pathway released MPIO label that could be taken up by surrounding microglia. Overall, the authors concluded that direct in vivo labeling of stem or progenitor cells is a technique that would allow for novel studies on cell migration and stem cell behavior. $^{40}$

\section{LIMITATIONS}

Superparamagnetic iron oxide is a passive contrast agent, in that the agent will produce contrast in MR images regardless of location, cell type, or cell viability. This leads to the potential for nonspecific findings, because residual label from nonviable cells may not be distinguishable from label contained within viable cells. Residual label can be taken up by macrophages and microglia and may contaminate MR images. ${ }^{31,40}$ Careful analysis and control experiments must be applied to ensure that the signal corresponds to and resides within the cell population of interest. Active MRI contrast agents, agents that only produce signal when within viable cells or specific cell types, will greatly improve MR-based cell transplantation studies. Preliminary work has been completed with active agents; however, the sensitivity of these agents for single-cell imaging remains to be established. ${ }^{41}$

Furthermore, cell division can have a negative effect on the ability to track cells. As the implanted cell population divides, the label is diluted when passed to daughter cells. It has been shown that cell proliferation can result in a dilution of the label and a decline in signal intensity. ${ }^{36}$ An alternative strategy that may help overcome this limitation is to genetically transduce a cell with a reporter gene that can produce superparamagnetic material. This novel concept has had some preliminary success using the ferritin gene. ${ }^{42,43}$ Overexpression of this gene leads to accumulation of endogenous iron. With a viral vector used for ferritin gene delivery, cells overexpressing ferritin could produce contrast on $\mathrm{T} 2$ and $\mathrm{T} 2 *$ sequences. This effect was also demonstrated in vivo following ferritin gene delivery to the mouse brain.

Many questions remain to be answered with respect to this alternative approach, particularly whether this approach will be useful for imaging of individual transplanted or host cells, and whether genetic alteration significantly affects the behavior of transplanted or endogenous cell populations.

\section{CONCLUSIONS}

Progress in developing cellular transplantation strategies for human clinical therapies has been somewhat hampered by the limitations inherent in conventional animal experimental methods. Conventional animal cell transplantation studies rely on the use of labels or tracer agents for cell identification in postmortem analyses. This labeling process can be achieved through the expression of reporter genes such as $\beta$-galactosidase, or through the intracellular accumulation of dyes that allow for cell-graft identification during conventional histology. Certain fundamental questions are extremely difficult, if not impossible, to address using these techniques.

For example, to determine the biodistribution of a cell graft, large parts of the target tissue must be sectioned and evaluated histologically. Information concerning quantitative engraftment rates, 3D spatial relationships, 
and dynamic information such as migration may be exceedingly difficult to obtain. In addition, within-subject, prospective analyses are of course not possible in studies requiring sacrifice and histology. The field of noninvasive cellular imaging has the potential to obviate many of the limitations posed by conventional histology, and to hasten human application of cellular transplantation therapies.

The application of superparamagnetic iron oxides to cellular imaging has allowed for small numbers and even single cells to be detected noninvasively. Studies using this technology have already begun to elucidate some of the basic mechanisms of transplanted and host cell population biology. This is a relatively young field, and the applications of this technology continue to increase. Further research can continue to expand and refine these techniques for potential human clinical use in studies of transplanted and endogenous cell populations.

\section{REFERENCES}

1. Rapalino O, Lazarov-Spiegler O, Agranov E, et al. Implantation of stimulated homologous macrophages results in partial recovery of paraplegic rats. Nat Med 1998;4:814-821.

2. Dunning MD, Lakatos A, Loizou L, et al. Superparamagnetic iron oxide-labeled Schwann cells and olfactory ensheathing cells can be traced in vivo by magnetic resonance imaging and retain functional properties after transplantation into the CNS. J Neurosci 2004;24:9799-9810.

3. Anderson SA, Shukaliak-Quandt J, Jordan EK, et al. Magnetic resonance imaging of labeled T-cells in a mouse model of multiple sclerosis. Ann Neurol 2004;55:654-659.

4. Bulte JW, Douglas T, Witwer B, et al. Magnetodendrimers allow endosomal magnetic labeling and in vivo tracking of stem cells. Nat Biotechnol 2001;19:1141-1147.

5. Arbab AS, Bashaw LA, Miller BR, et al. Characterization of biophysical and metabolic properties of cells labeled with superparamagnetic iron oxide nanoparticles and transfection agent for cellular MR imaging. Radiology 2003;229:838-846.

6. Arbab AS, Jordan EK, Wilson LB, Yocum GT, Lewis BK, Frank JA. In vivo trafficking and targeted delivery of magnetically labeled stem cells. Hum Gene Ther 2004;15:351-360.

7. Frank JA, Miller BR, Arbab AS, et al. Clinically applicable labeling of mammalian and stem cells by combining superparamagnetic iron oxides and transfection agents. Radiology 2003;228:480-487.

8. Hayakawa J, Migita M, Ueda T, Shimada T, Fukunaga Y. Generation of a chimeric mouse reconstituted with green fluorescent protein-positive bone marrow cells: a useful model for studying the behavior of bone marrow cells in regeneration in vivo. Int $\mathrm{J} \mathrm{He}-$ matol 2003;77:456-462.

9. Bulte JW, Zhang S, van Gelderen P, et al. Neurotransplantation of magnetically labeled oligodendrocyte progenitors: magnetic resonance tracking of cell migration and myelination. Proc Natl Acad Sci U S A 1999;96:15256-15261.

10. Josephson L, Tung CH, Moore A, Weissleder R. High-efficiency intracellular magnetic labeling with novel superparamagnetic-Tat peptide conjugates. Bioconjug Chem 1999;10:186-191.

11. Lewin $\mathrm{M}$, Carlesso N, Tung $\mathrm{CH}$, et al. Tat peptide-derivatized magnetic nanoparticles allow in vivo tracking and recovery of progenitor cells. Nat Biotechnol 2000;18:410-414.

12. Weissleder R, Elizondo G, Wittenberg J, Rabito CA, Bengele HH, Josephson L. Ultrasmall superparamagnetic iron oxide: characterization of a new class of contrast agents for MR imaging. Radiology 1990;175:489-493.
13. Shen T, Weissleder R, Papisov M, Bogdanov A Jr, Brady TJ. Monocrystalline iron oxide nanocompounds (MION): physicochemical properties. Magn Reson Med 1993;29:599-604.

14. Moore A, Grimm J, Han B, Santamaria P. Tracking the recruitment of diabetogenic CD8 + T-cells to the pancreas in real time. Diabetes 2004;53:1459-1466.

15. Jirak D, Kriz J, Herynek V, et al. MRI of transplanted pancreatic islets. Magn Reson Med 2004;52:1228-1233.

16. Bulte JW, Duncan ID, Frank JA. In vivo magnetic resonance tracking of magnetically labeled cells after transplantation. J Cereb Blood Flow Metab 2002;22:899-907.

17. Brown MA, Semelka RC. MRI: basic principles and applications, 2nd ed. New York: Wiley-Liss, 1999.

18. Wang YX, Hussain SM, Krestin GP. Superparamagnetic iron oxide contrast agents: physicochemical characteristics and applications in MR imaging. Eur Radiol 2001;11:2319-2331.

19. Wood ML, Hardy PA. Proton relaxation enhancement. J Magn Reson Imaging 1993;3:149-156.

20. Schoepf U, Marecos EM, Melder RJ, Jain RK, Weissleder R. Intracellular magnetic labeling of lymphocytes for in vivo trafficking studies. Biotechniques 1998;24:642-646, 648-651.

21. Dodd $\mathrm{CH}$, Hsu HC, Chu WJ, et al. Normal T-cell response and in vivo magnetic resonance imaging of $\mathrm{T}$ cells loaded with HIV transactivator-peptide-derived superparamagnetic nanoparticles. J Immunol Methods 2001;256:89-105.

22. Dennig J, Duncan E. Gene transfer into eukaryotic cells using activated polyamidoamine dendrimers. J Biotechnol 2002;90:339347.

23. Shah DS, Sakthivel T, Toth I, Florence AT, Wilderspin AF. DNA transfection and transfected cell viability using amphipathic asymmetric dendrimers. Int J Pharm 2000;208:41-48.

24. Yoo H, Juliano RL. Enhanced delivery of antisense oligonucleotides with fluorophore-conjugated PAMAM dendrimers. Nucleic Acids Res 2000;28:4225-4231.

25. Hudde T, Rayner SA, Comer RM, et al. Activated polyamidoamine dendrimers, a non-viral vector for gene transfer to the corneal endothelium. Gene Ther 1999;6:939-943.

26. Kukowska-Latallo JF, Bielinska AU, Johnson J, Spindler R, Tomalia DA, Baker JR Jr. Efficient transfer of genetic material into mammalian cells using Starburst polyamidoamine dendrimers. Proc Natl Acad Sci U S A 1996;93:4897-4902.

27. Bulte JW, Douglas T, Witwer B, et al. Monitoring stem cell therapy in vivo using magnetodendrimers as a new class of cellular MR contrast agents. Acad Radiol 2002;9 Suppl 2:S332-S335.

28. Walter GA, Cahill KS, Huard J, et al. Noninvasive monitoring of stem cell transfer for muscle disorders. Magn Reson Med 2004; 51:273-277.

29. Frank JA, Zywicke H, Jordan EK, et al. Magnetic intracellular labeling of mammalian cells by combining (FDA-approved) superparamagnetic iron oxide MR contrast agents and commonly used transfection agents. Acad Radiol 2002;9 Suppl 2: S484-S487.

30. Kalish H, Arbab AS, Miller BR, et al. Combination of transfection agents and magnetic resonance contrast agents for cellular imaging: relationship between relaxivities, electrostatic forces, and chemical composition. Magn Reson Med 2003;50:275-282.

31. Cahill KS, Gaidosh G, Huard J, Silver X, Byrne BJ, Walter GA Noninvasive monitoring and tracking of muscle stem cell transplants. Transplantation 2004;78:1626-1633.

32. Dodd SJ, Williams M, Suhan JP, Williams DS, Koretsky AP, Ho C. Detection of single mammalian cells by high-resolution magnetic resonance imaging. Biophys J 1999;76:103-109.

33. Hoehn M, Kustermann E, Blunk J, et al. Monitoring of implanted stem cell migration in vivo: a highly resolved in vivo magnetic resonance imaging investigation of experimental stroke in rat. Proc Natl Acad Sci U S A 2002;99:16267-16272.

34. Foster-Gareau P, Heyn C, Alejski A, Rutt BK. Imaging single mammalian cells with a $1.5 \mathrm{~T}$ clinical MRI scanner. Magn Reson Med 2003;49:968-971.

35. Heyn C, Ronald JA, Mackenzie LT, et al. In vivo magnetic resonance imaging of single cells in mouse brain with optical validation. Magn Reson Med 2006;55:23-29. 
36. Hinds KA, Hill JM, Shapiro EM, et al. Highly efficient endosomal labeling of progenitor and stem cells with large magnetic particles allows magnetic resonance imaging of single cells. Blood 2003; 102:867-872

37. Shapiro EM, Skrtic S, Koretsky AP. Sizing it up: cellular MRI using micron-sized iron oxide particles. Magn Reson Med 2005; 53:329-338.

38. Shapiro EM, Skrtic S, Sharer K, Hill JM, Dunbar CE, Koretsky AP. MRI detection of single particles for cellular imaging. Proc Natl Acad Sci U S A 2004;101:10901-10906.

39. Shapiro EM, Sharer K, Skrtic S, Koretsky AP. In vivo detection of single cells by MRI. Magn Reson Med 2006;55:242-249.
40. Shapiro EM, Gonzalez-Perez O, Manuel Garcia-Verdugo J, Alvarez-Buylla A, Koretsky AP. Magnetic resonance imaging of the migration of neuronal precursors generated in the adult rodent brain. Neuroimage 2006;32:1150-1157.

41. Louie AY, Huber MM, Ahrens ET, et al. In vivo visualization of gene expression using magnetic resonance imaging. Nat Biotechnol 2000;18:321-325.

42. Cohen B, Ziv K, Plaks V, et al. MRI detection of transcriptional regulation of gene expression in transgenic mice. Nat Med 2007;13:498-503.

43. Genove G, DeMarco U, Xu H, Goins WF, Ahrens ET. A new transgene reporter for in vivo magnetic resonance imaging. Nat Med 2005;11:450-454. 